\title{
Molecular evolution of the cytochrome c oxidase subunit 5A gene in primates
}

\author{
Monica Uddin ${ }^{\dagger 1}$, Juan C Opazo ${ }^{\dagger 1,2}$, Derek E Wildman ${ }^{1,3,4}$, \\ Chet C Sherwood ${ }^{5}$, Patrick R Hof ${ }^{6}$, Morris Goodman ${ }^{1,7}$ and \\ Lawrence I Grossman*1
}

\begin{abstract}
Address: ${ }^{1}$ Center for Molecular Medicine and Genetics, Wayne State University School of Medicine, Detroit MI 48201, USA, ${ }^{2}$ Instituto de Ecología y Evolución, Facultad de Ciencias, Universidad Austral de Chile, Casilla 567, Valdivia, Chile, ${ }^{3}$ Perinatology Research Branch, National Institute of Child Health and Development, National Institutes of Health, Bethesda, MD 20892, USA, ${ }^{4}$ Department of Obstetrics and Gynecology, Wayne State University School of Medicine and Hutzel Women's Hospital, Detroit, MI 48201, USA, ${ }^{5}$ Department of Anthropology, The George Washington University, Washington DC 20052, USA, 'Department of Neuroscience, Mount Sinai School of Medicine, New York, NY 10029, USA and ${ }^{7}$ Department of Anatomy and Cell Biology, Wayne State University School of Medicine, Detroit MI 48201, USA
\end{abstract}

Email: Monica Uddin - muddin@med.wayne.edu; Juan C Opazo - jopazo2@unlnotes.unl.edu; Derek E Wildman - dwildman@genetics.wayne.edu; Chet C Sherwood - sherwood@gwu.edu; Patrick R Hof - patrick.hof@mssm.edu; Morris Goodman - mgoodwayne@aol.com; Lawrence I Grossman* - l.grossman@wayne.edu

* Corresponding author †Equal contributors

Published: 15 January 2008

BMC Evolutionary Biology 2008, 8:8 doi:10.1/86/147|-2/48-8-8
Received: 10 May 2007

Accepted: 15 January 2008

This article is available from: http://www.biomedcentral.com/I47I-2/48/8/8

(c) 2008 Uddin et al; licensee BioMed Central Ltd.

This is an Open Access article distributed under the terms of the Creative Commons Attribution License (http://creativecommons.org/licenses/by/2.0), which permits unrestricted use, distribution, and reproduction in any medium, provided the original work is properly cited.

\begin{abstract}
Background: Many electron transport chain (ETC) genes show accelerated rates of nonsynonymous nucleotide substitutions in anthropoid primate lineages, yet in non-anthropoid lineages the ETC proteins are typically highly conserved. Here, we test the hypothesis that COX5A, the ETC gene that encodes cytochrome $c$ oxidase subunit 5A, shows a pattern of anthropoidspecific adaptive evolution, and investigate the distribution of this protein in catarrhine brains.

Results: In a dataset comprising 29 vertebrate taxa, including representatives from all major groups of primates, there is nearly $100 \%$ conservation of the COX5A amino acid sequence among extant, non-anthropoid placental mammals. The most recent common ancestor of these species lived about 100 million years (MY) ago. In contrast, anthropoid primates show markedly elevated rates of nonsynonymous evolution. In particular, branch site tests identify five positively selected codons in anthropoids, and ancestral reconstructions infer that substitutions in these codons occurred predominantly on stem lineages (anthropoid, ape and New World monkey) and on the human terminal branch. Examination of catarrhine brain samples by immunohistochemistry characterizes for the first time COX5A protein distribution in the primate neocortex, and suggests that the protein is most abundant in the mitochondria of large-size projection neurons. Real time quantitative PCR supports previous microarray results showing COX5A is expressed in cerebral cortical tissue at a higher level in human than in chimpanzee or gorilla.

Conclusion: Taken together, these results suggest that both protein structural and gene regulatory changes contributed to COX5A evolution during humankind's ancestry. Furthermore, these findings are consistent with the hypothesis that adaptations in ETC genes contributed to the emergence of the energetically expensive anthropoid neocortex.
\end{abstract}




\section{Background}

The electron transport chain (ETC) genes encode essential components of the mitochondrial machinery that carries out aerobic energy production. Since the integrity of this machinery must be maintained to sustain life, the mammalian ETC proteins are generally evolutionarily conserved. Among primates, however, the ETC genes show marked upsurges in the rate of nonsynonymous (aminoacid changing) substitutions [1-17]. These upsurges, indicative of adaptive evolution, have not occurred randomly; rather, they have occurred within anthropoid primates [9], a clade that includes New and Old World monkeys and apes, including humans. Life history and morphological features characteristic of this clade include a relatively enlarged cerebral cortex, prolonged intrauterine fetal development followed postnatally by prolonged dependency of the young on nurturing adults, and longer lifespan. The aerobic demand of these features makes it plausible that they have acted as selective pressures during anthropoid evolution, producing adaptive amino acid and possibly regulatory changes in aerobic energy producing ETC genes.

Previous work suggested that among ETC genes, COX5A, the nuclear gene encoding cytochrome $c$ oxidase subunit 5A, may provide a striking example of adaptive evolution. Initial characterization of subunit $5 \mathrm{~A}$ in human revealed a high degree of conservation with the corresponding subunit in cow [18]. In a subsequent bioinformatics study, Schmidt et al. [13] found that, among COX5A protein (COX5Ap) sequences from mammals, the human orthologue (the only primate representative in that dataset) differed by five amino acid residues from mouse, rat, cow and pig, all of which had identical amino acid sequences. Here, with newly generated COX5A coding region nucleotide sequences and bioinformatically extracted orthologues from publicly available whole genome sequences, we tested whether this ETC gene shows the pattern of anthropoid-specific adaptive evolution observed in 14 other ETC proteins $[9,10]$. We did so within the context of a robust statistical framework, employing the PAML package [19] to determine: 1 ) whether the anthropoid clade has a different ratio of nonsynonymous/synonymous substitution rates (i.e., omega value) when compared to other placental mammals and 2) whether particular sites in COX5Ap show the signature of positive selection within the anthropoid clade.

In addition to these sequence-based analyses, we investigated patterns of COX5A expression and localization in catarrhine brains using immunohistochemistry and quantitative real time PCR (qRT-PCR) techniques. Finally, we identified the anthropoid lineages on which changes in COX5Ap residues that interact with other cytochrome $c$ oxidase (COX) subunits occurred. Based on these results, we explored the hypothesis that COX5A evolution in humankind's ancestry involved both protein structural and gene regulatory changes, and that the anthropoidspecific evolution observed in ETC proteins represents an adaptive response to the increased energy demands of an enlarged neocortex.

\section{Results and Discussion}

Complete COX5A nucleotide coding region and deduced amino acid sequences encoding the mature peptide were analysed in 26 vertebrate species, including newly generated data from 14 primate species: common chimpanzee (Pan troglodytes), bonobo (Pan paniscus), gorilla (Gorilla gorilla), orangutan (Pongo pygmaeus), red-cheeked gibbon (Nomascus gabriellae), siamang (Symphalangus syndactylus), mantled guereza (Colobus guereza), olive baboon (Papio anubis), pygmy marmoset (Callithrix pygmaea), whitelipped tamarin (Saguinus labiatus), white-eared titi monkey (Callicebus donacophilus), slow loris (Nycticebus coucang), brown greater galago (Otolemur crassicaudatus) and brown lemur (Eulemur fulvus); and one marsupial, the parma wallaby (Macropus parma). Partial sequences were obtained for three additional taxa (cat, rabbit, and chicken). Apart from a single amino acid difference in the elephant sequence, the protein sequence alignment showed no amino acid changes from the last common ancestor of placental mammals to present-day nonanthropoids, in a sampling that includes representatives from every major placental mammalian clade except Xenarthra (Fig. 1). Interestingly, the elephant is similar to anthropoids in that both share the aerobically demanding feature of lengthy gestation. Within anthropoids, 13 amino acid replacements were inferred by all ancestral state reconstruction methods, and eight of these occurred on the human lineage from the last common ancestor (LCA) of haplorhines (tarsier and anthropoids) (Fig. 2).

\section{Evidence for adaptive evolution}

Results from the model-based codeml analyses confirm that COX5A omega ratios vary among lineages in vertebrates. Specifically, the statistical model assuming one omega value for all branches (i.e., the one ratio model) had a likelihood value of -1431.66 , with an omega value of 0.048. The model that assumes an independent omega value for each branch (i.e., the free ratio model) had a likelihood value of -1388 . According to the likelihood ratio test, the free ratio model fit the data significantly better than the one ratio model $(\mathrm{p}<0.01)$. The inferred omega values and the numbers of nonsynonymous and synonymous substitutions under the free ratio model are presented in Fig. 1. In addition, the model that segregates anthropoids from non-anthropoid placental mammals fit the data significantly better than the model that assigned the same omega value to all placental mammals $\left(\mathrm{p}<10^{-}\right.$ $\left.{ }^{5}\right)$, indicating that the ratio of nonsynonymous/synony- 


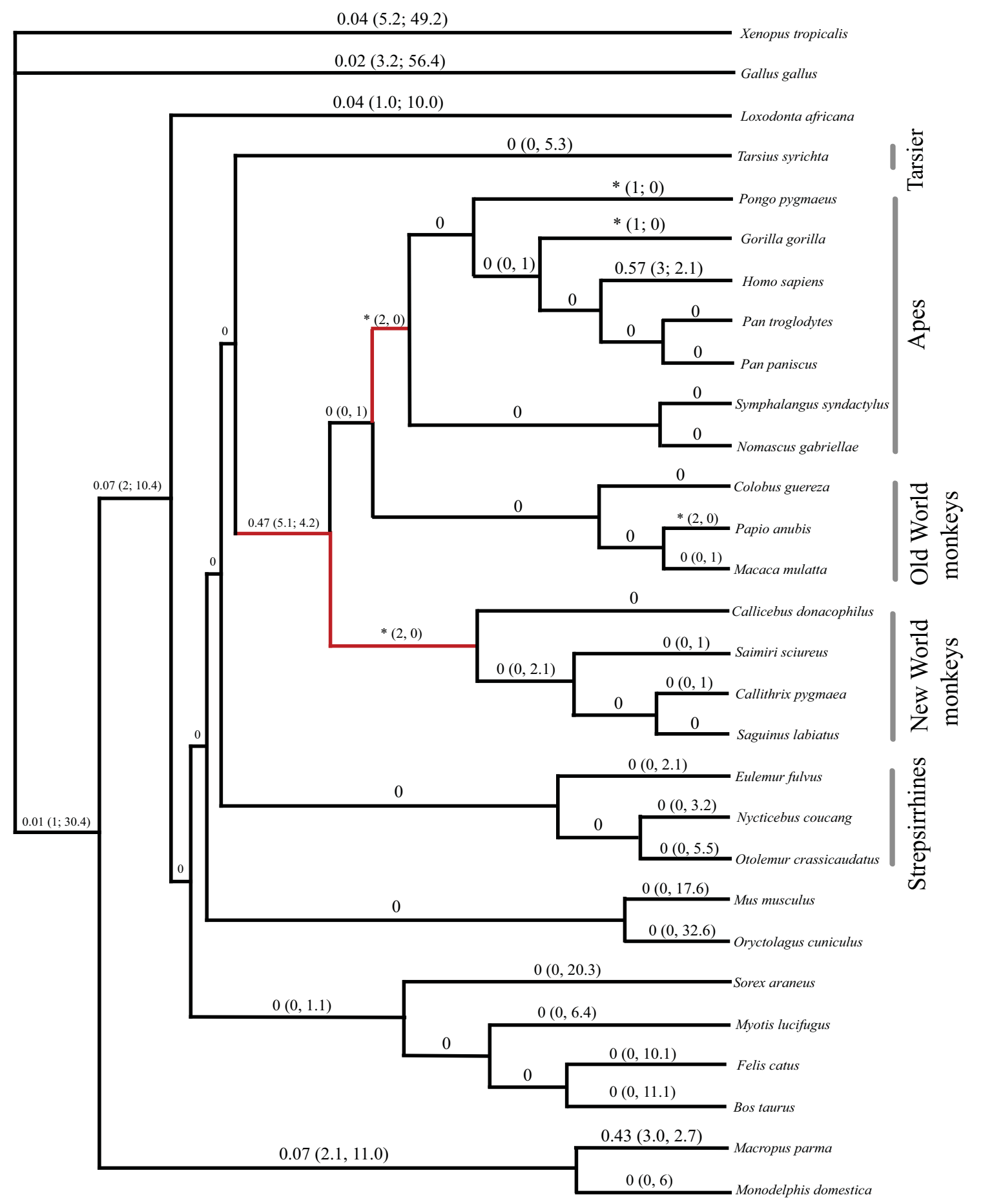

Figure I

Phylogenetic tree depicting the omega ratios and (number of nonsynonymous and synonymous substitutions) for COX5A in each branch of the tree, as estimated by PAML under the free ratio (MI) model. " 0 " and "*" indicate, respectively, lineages on which the number of nonsynonymous and synonymous changes, as well as $\mathrm{dN}$ and $\mathrm{dS}$, were estimated to be effectively 0 (i.e., $<0.00004$ ); and lineages on which the number of synonymous changes was estimated to be 0 (i.e., omega is undefined). The anthropoid, New World monkey and ape stems, indicated in red, depict a classic pattern of positive selection [22]: markedly increased $\mathrm{dN}$ relative to $\mathrm{dS}$ followed by descendant lineages with a markedly decreased $\mathrm{dN}$ relative to ds. 


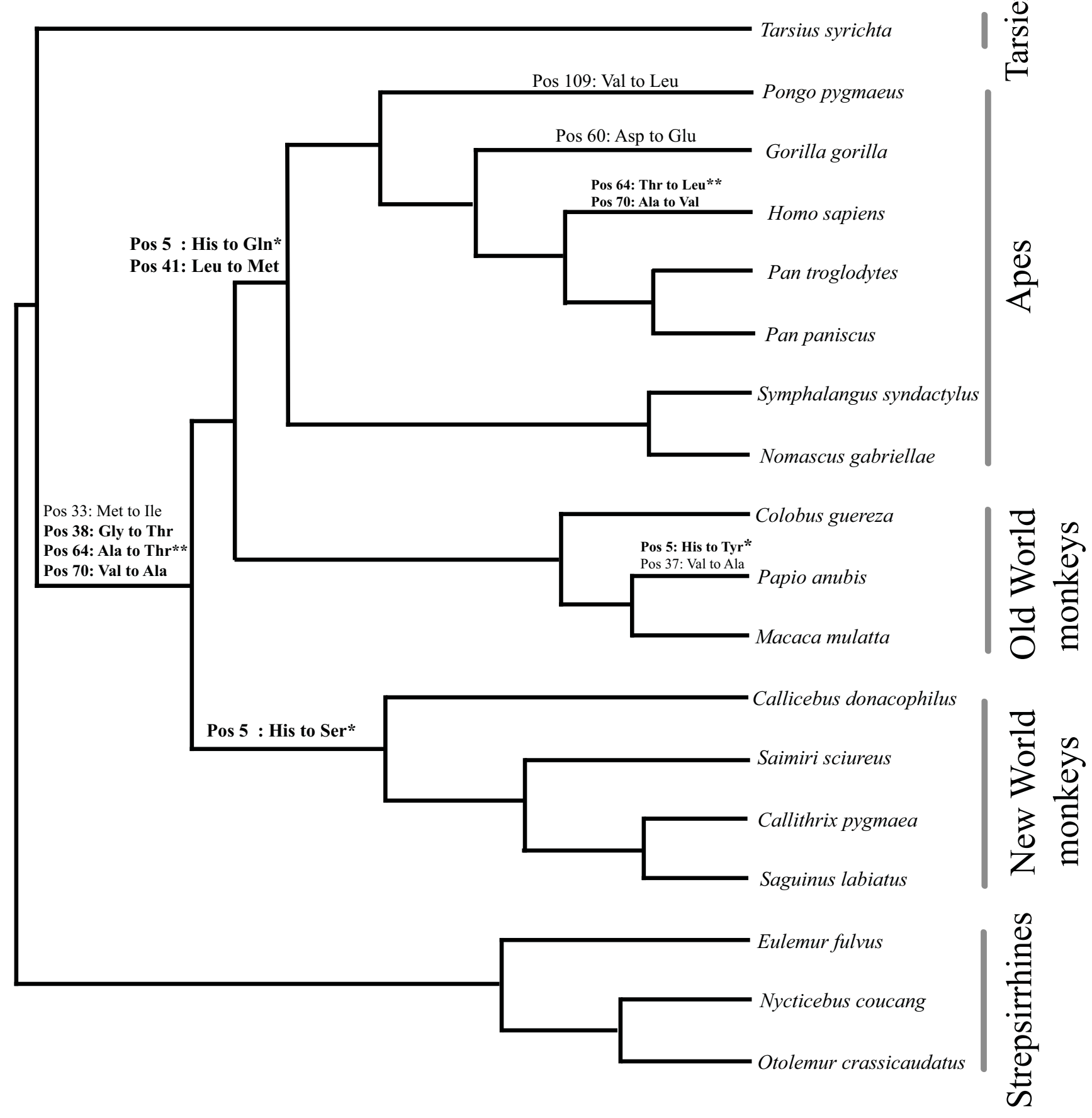

\section{Figure 2}

Phylogenetic tree depicting amino acid replacements inferred in COX5Ap within the anthropoid clade determined via maximum parsimony (ACCTRAN and DELTRAN) as implemented in PAUP* [5I], and the codon substitution model [52] implemented in PAML 3.I5. Non-prosimian outgroup species are not shown. Bold type indicates positively selected amino acids identified via the branch site test using PAML (Table I). * Amino acid replacements in which the charge has changed, ** amino acid replacements in which the polarity has changed. 
mous substitution rates differs in this clade; in particular, the omega value inferred for anthropoids is 52 times greater than that inferred for non-anthropoid placental mammals (0.431 vs. 0.008 ; Table 1$)$.

Despite this elevated ratio in anthropoids, we note that the overall ratio is still less than one. This result, however, is based on the assumption that all codons are under the same selective pressure and thus share the same underlying omega value. Because this assumption is "grossly unrealistic" [20], and because important changes in function can result from a few key amino acid changes that do not elevate the overall omega ratio above one [21], we conducted a branch-site test of adaptive evolution. Here, the Anthropoidea as a total group was fixed as the foreground lineage, i.e., the branches on which there was permitted a class of sites with an omega $>1$. Results from this test provide evidence of positive selection on particular sites during anthropoid evolution: the model allowing a class of sites with an omega higher than one (i.e., positive selection) fit the data significantly better than an alternative model that fixed omega at one for this same site class $(\mathrm{p}<0.002)$. Specifically, five sites were identified as positively selected, four of which had a posterior probability > 0.95 (Table 1). Furthermore, we note that despite the many amino acid replacements inferred to have occurred throughout the Anthropoidea (see below), the five positively selected sites show changes occurring predominantly on stem lineages (anthropoid, ape, and New World monkey) and on the human terminal branch. The localization of these particular sites to these three stem lineages, combined with the M1 analysis (Fig. 1) depicting a classic sequence [22] of markedly increased $\mathrm{dN}$ relative to $\mathrm{dS}$, followed by descendant lineages with a markedly decreased $\mathrm{dN}$ relative to $\mathrm{dS}$, presents a pattern strongly indicative of positive selection.
Ancestral state reconstruction methods confirm and extend results obtained by the codeml analysis. Among the five sites identified as positively selected by the branch site test, four were inferred to have changed on more than one anthropoid lineage, and three were inferred to have changed on the anthropoid stem (Fig. 2). In addition, all three ancestral state reconstruction methods inferred a fourth amino acid change on the anthropoid stem that was not identified by the branch site test: a methionine to isoleucine at residue 33 . It is tempting to speculate that this replacement may have acted as a "permissive" change that then allowed the selection of the other three amino acid changes occurring on this and perhaps other descendant anthropoid branch(es). This sequence of events was recently demonstrated in an elegant study by Ortlund et al. [23] in their investigation of cortisol-sensitive glucocorticoid receptor (GR) evolution in vertebrates. Using a combination of structural, phylogenetic and functional analyses of ancestral corticosteroid receptors, the authors identified a small set of permissive amino acid changes that stabilized specific elements of the protein. In conjunction with additional changes that diminished the receptor's sensitivity to aldosterone and increased its sensitivity to cortisol, these permissive changes allowed an additional and subsequent set of function-switching amino acid changes that conferred a fully GR-like phenotype that is sensitive only to cortisol. With respect to COX5Ap, verification of this hypothesis in future studies would require additional, fine-tuned functional data regarding the effects of each of the observed replacements alone and in concert on the activity of the reconstructed protein.

\section{Expression and localization of COX5A}

Quantitative RT-PCR results showed that relative COX5A gene expression levels in the anterior cingulate cortex vary among human, chimpanzee, gorilla, and macaque (Fig. 3). Specifically, human and macaque showed similar gene

Table I: Parameter estimates and likelihood values under branch and branch-site models.

\begin{tabular}{|c|c|c|c|c|}
\hline Model & $P$ & likelihood & Parameters estimated & Positively selected sites \\
\hline \multicolumn{5}{|l|}{ Branch Models } \\
\hline$I \omega$ & 1 & $-|43| .66$ & $\omega$ all branches $=0.048$ & $\mathrm{~N} / \mathrm{A}$ \\
\hline $2 \omega$ & 2 & $-|43| .4 \mid$ & $\omega$ placental mammals $=0.054 ;$ background $=0.04$ & N/A \\
\hline $3 \omega$ & 3 & -1408.70 & $\begin{array}{l}\omega \text { anthropoid }=0.43 \mathrm{I} ; \omega \text { other placentals }=0.008 ; \omega \\
\text { background }=0.042\end{array}$ & N/A \\
\hline Free Ratio & 55 & -1388.00 & $\omega$ estimated independently for each branch (see Fig. I) & N/A \\
\hline \multicolumn{5}{|l|}{ Branch-site models } \\
\hline Model A & 4 & -1399.23 & $\begin{array}{l}P_{0}=0.90 ; P_{1}=0.012 ; P_{(2 a+2 b)}=0.085 ; \omega_{0}=0.021 ; \omega_{1}=1 ; \\
\text { background } \omega_{2 a}=0.021 ; \omega_{2 b}=1 ; \text { foreground, } \omega_{2 a}=\omega_{2 b}=4.61\end{array}$ & $\begin{array}{l}5(1.00) ; 38(0.99) ; 41(0.53) ; 64 \\
(1.00) ; 70(0.97)\end{array}$ \\
\hline Model A (omega fixed) & 3 & -1403.73 & $\begin{array}{l}P_{0}=0.80 ; P_{1}=0.011 ; P_{(2 a+2 b)}=0.192 ; \omega_{0}=0.02 ; \omega_{1}=1 ; \\
\text { background } \omega_{2 a}=0.02 ; \omega_{2 b}=1 ; \text { foreground, } \omega_{2 a}=\omega_{2 b}=1\end{array}$ & Not allowed \\
\hline
\end{tabular}

P, number of parameters. Numbers in parentheses represent the posterior probability of each amino acid listed to be in the class of sites under positive selection. 


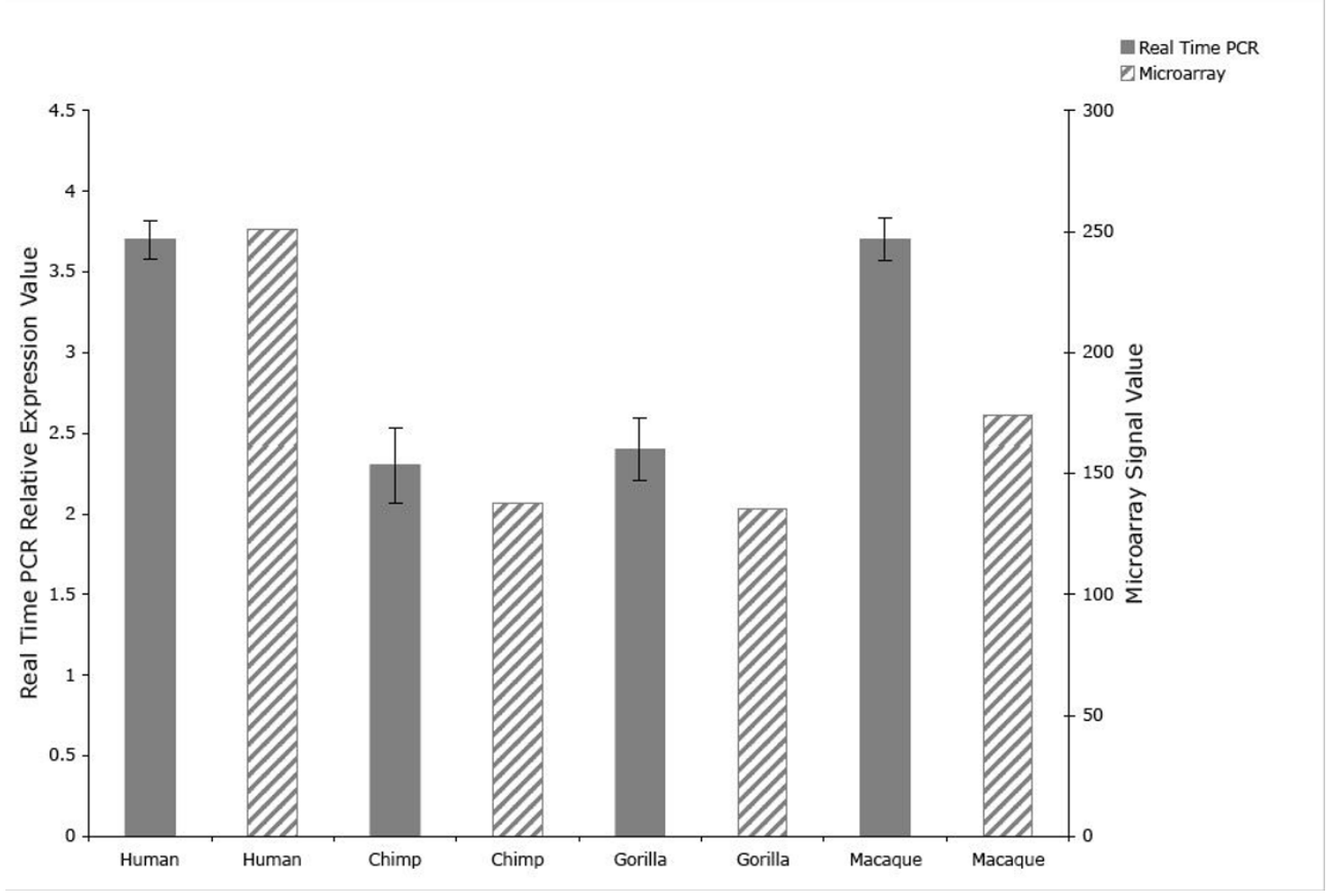

Figure 3

COX5A Expression levels as determined by quantitative RT-PCR (solid bars) and microarray signal values (hatched bars). qRTPCR expression levels are expressed in relative terms, with samples compared to a calibrator amplified from human reference total RNA and represented as species means $( \pm$ SEM). Samples were tested in triplicate, providing 9 total measurements for human and macaque and three total measurements for chimpanzee and gorilla. Microarray expression levels were as determined in [24].

expression levels, as did chimpanzee and gorilla, and the first pair of species showed higher levels of expression than the second pair (Fig. 3). This pattern confirms the trend previously suggested by microarray analysis [24], and represents a more robust dataset from which to make such observations: unlike the previous experiment, which involved hybridizing non-human samples to a humanspecific microarray, these qRT-PCR results are not confounded by sequence mismatches between sample and microarray chip. Specifically, the COX5A expression results reported by Uddin et al. [24] were based on an Affymetrix probe set (203663_s_at) that shows $0.8 \%$ difference with the published chimpanzee genome sequence [25] and 7.3\% difference with the published rhesus macaque sequence [26]. Thus, the present results suggest that COX5A has undergone not only coding region sequence evolution, but also regulatory evolution in anthropoid primates. This hypothesis is supported by the observation of primate COX5A expression patterns in other tissues that differ from those reported here: in microarray experiments performed on fibroblast cell lineages, humans showed a lower COX5A expression level than did bonobos or gorillas, and comparison of the human and bonobo expression levels showed a significant difference [27]. Nevertheless, without additional samples from additional chimpanzees and gorillas, the possibility that our results are due to interindividual differences within these species and/or age-related differences in gene expression profiles cannot formally be ruled out.

Among macaques, gorillas, chimpanzees, and humans, the localization of COX5Ap as revealed by immunohistochemistry showed a punctate staining pattern that is consistent with the morphology of mitochondria (Fig. 4, data from gorilla not shown). Similar staining patterns were obtained in all species with both monoclonal and polyclonal antibodies. As evident by colocalization with microtubule-associated protein 2 (MAP2) and neuronspecific nuclear protein (NeuN), COX5Ap was particularly enriched in the cytoplasm of the soma and proximal apical dendrite of neurons, especially large pyramidal cells. We also observed diffuse COX5Ap-immunoreactive puncta throughout the neuropil, which corresponds to 

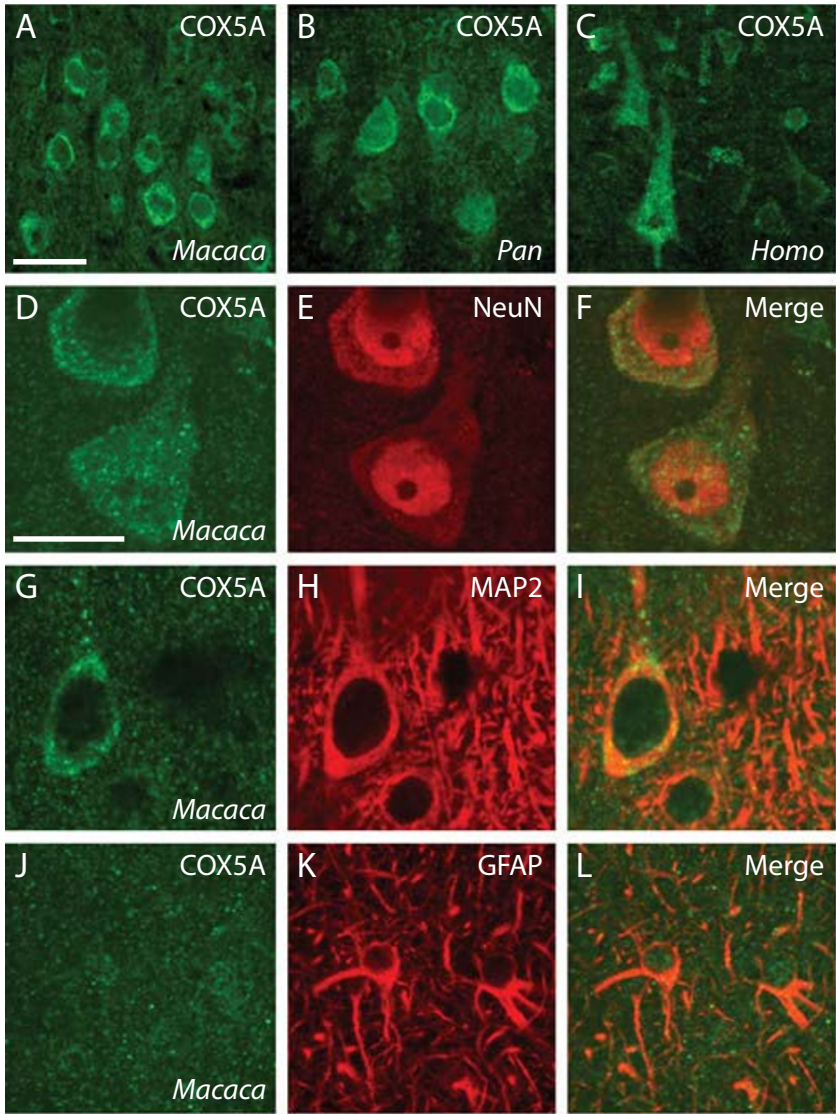

Figure 4

Immunohistochemical staining of COX5A protein in the dorsolateral prefrontal cortex. The distribution of staining using a monoclonal antibody against COX5Ap in macaque monkey (A), chimpanzee (B), and human (C). Panels D-L show double label immunostaining from macaque monkey. Images in A-I are taken from layer III. Images in J-L are taken from layer I. Scale bar in panel $A=50 \mu \mathrm{m}$ applies to A-C. Scale bar in D = $25 \mu \mathrm{m}$ applies to D-L.

the space occupied by glia, dendritic processes, axons, and synapses. Double immunostaining against glial fibrillary acidic protein (GFAP) demonstrated that astrocytes do not show high levels of COX5Ap. Indeed, $95.3 \%$ of cells that are enriched with COX5Ap also contain the neuronspecific protein NeuN in macaques, as estimated by optical disector counting. The white matter did not display a high density of COX5Ap staining.

These results suggest that COX5Ap is most abundant in the mitochondria of large-size projection neurons of the neocortex. It is noteworthy that neocortical enlargement in primates is associated with increasing numbers of large pyramidal neurons that are intensely neurofilament $\mathrm{H}$ protein-immunoreactive, have long-range projecting axons, and are presumably metabolically costly [28]. To understand more completely the functional role of COX5A in neocortical circuits and the possible consequences of positive selection on this gene, it will be necessary to define further the phenotype and distribution of cells in the brain that show high levels of expression for COX5Ap using strategies such as laser microcapture dissection combined with qRT-PCR or mass spectroscopy proteomics in future studies.

\section{Role of accelerated COX evolution}

As we have demonstrated, changes in COX5Ap are rare among placental mammals outside the primate clade. Among four positively selected sites identified by the branch site test with posterior probabilities $>0.95$, two showed changes in their physicochemical properties: residue 5, which changed from positively charged to neutral on the ape and New World monkey stems and on the baboon terminal (Fig. 2); and residue 64, which changed from nonpolar to polar in the anthropoid stem and from polar to nonpolar on the human terminal. Interestingly, the charge neutralizations are parallel to those observed at the binding site for cytochrome $c$ on COX in anthropoids, where the binding site changes reduced the electrostatic interaction between the docked molecules [15]. Of note, one amino acid change on the human terminal at position 70 (Ala to $\mathrm{Val}$ ) is a reversal of a change on the anthropoid stem (Fig. 2).

Although the role of the inferred amino acid changes in COX5Ap is currently unknown, it is noteworthy that the changes in this protein are located in residues that are in proximity to other, physically close, COX subunits (Table 2 ). Of the five positively selected sites identified by the branch site test in COX5Ap, seven replacements within 10 $\AA$ of these sites have occurred in COX2p and COX4p during anthropoid evolution. Examination of the nature of the changes suggests there has been an electrostatic change such that the interaction between position 5 of COX5Ap and residues 52 and 57 of COX2p has been neutralized; in addition, residues 55 and 56 of this subunit have changed from polar to nonpolar. Furthermore, the potentially interacting, altered residues of COX4p (22 and 63) have changed from neutral to positive charge (Fig. 2 and Table 2), forming a positive patch. Of particular interest is that COX4p residue 22, which has changed from Tyr to His, is adjacent to the proposed ATP binding site that includes COX4p 20 [29].

An additional consequence of the location of COX5Ap in close contact to the nucleotide-binding domain of COX4p is potential regulation by 3,5-diiodothyronine $\left(\mathrm{T}_{2}\right)$, a thyroid hormone produced when the iodothyronine deiodinase D3 acts over thyroxine $\left(\mathrm{T}_{4}\right)$ [30]. $\mathrm{T}_{2}$ has biological activity stimulating mitochondrial respiration by nuclear independent pathways $[30,31]$ and is known to bind to 
Table 2: Inferred amino acid replacements in COX2p and COX4p that occur in proximity to positively selected sites in COX5Ap among anthropoid primate stem lineages.

\begin{tabular}{|c|c|c|c|c|c|}
\hline \multicolumn{2}{|r|}{ COX5Ap } & \multicolumn{2}{|r|}{$\operatorname{cox} 2 p$} & \multicolumn{2}{|c|}{$\operatorname{cox} 4 p$} \\
\hline Residue & Lineage & Residue & Lineage & Residue & Lineage \\
\hline 5 ( $\mathrm{H}$ to $\mathrm{Q})$ & Ape stem & $52(\mathrm{H}$ to $\mathrm{N})$ & Catarrhine* stem & & \\
\hline \multirow[t]{5}{*}{$(H$ to $S)$} & New World monkey stem & $54(\mathrm{~S}$ to $\mathrm{N})$ & Catarrhine stem & & \\
\hline & & 55 (T to I) & Catarrhine stem & & \\
\hline & & $56(\mathrm{M}$ to $\mathrm{T})$ & Catarrhine stem & & \\
\hline & & $56(\mathrm{~T}$ to $\mathrm{S})$ & Large-bodied ape stem & & \\
\hline & & $57(\mathrm{D}$ to $\mathrm{N})$ & New World monkey stem & & \\
\hline \multirow[t]{5}{*}{38 (G to $T)$} & Anthropoid stem & $52(\mathrm{H}$ to $\mathrm{N})$ & Catarrhine stem & & \\
\hline & & $54(\mathrm{~S}$ to $\mathrm{N})$ & Catarrhine stem & & \\
\hline & & 55 (T to I) & Catarrhine stem & $22(\mathrm{Y}$ to $\mathrm{H})$ & Ape stem \\
\hline & & $56(\mathrm{M}$ to $\mathrm{T})$ & Catarrhine stem & & \\
\hline & & 56 (T to $S)$ & Large-bodied ape stem & & \\
\hline \multirow[t]{2}{*}{$4 I(L$ to $M)$} & Ape stem & $52(\mathrm{H}$ to $\mathrm{N})$ & Catarrhine stem & & \\
\hline & & $54(\mathrm{~S}$ to $\mathrm{N})$ & Catarrhine stem & & \\
\hline $64(\mathrm{~A}$ to $\mathrm{T})$ & Anthropoid stem & & & $63(\mathrm{Q}$ to $\mathrm{K})$ & Catarrhine stem \\
\hline $70(\mathrm{~V}$ to $\mathrm{A})$ & Anthropoid stem & & & $22(\mathrm{Y}$ to $\mathrm{H})$ & Ape stem \\
\hline
\end{tabular}

Interaction is taken as a distance $<10 \AA$ between residues.

*Catarrhines include Old World monkeys and apes, including humans

COX5Ap and abolish the allosteric inhibition of respiration at high ATP/ADP ratios [32]. More generally, triiodothyronine $\left(\mathrm{T}_{3}\right)$ thyroid hormone is considered to be a major regulator of mitochondrial activity [33] and has been shown to regulate the expression of a number of COX subunits, inducing functional increases in COX enzyme activity [34]. COX5A amino acid evolution may thus represent the product of selective pressures acting on the regulation of metabolism. In the context of this hypothesis, we note that thyroid hormones are known to play a critical role in early brain development [35-37] and that humans show evidence for a greater affinity of transthyretin and/or thyroxine-binding globulin for thyroid hormone when compared to chimpanzees [38]. Thus, it is plausible that the interaction of thyroid hormones with COX5Ap and other COX subunits may affect both the regulation of metabolism and the growth and development of important organ systems.

\section{Conclusion}

We have shown that COX5A has been subject to protein structural evolutionary changes in anthropoid primates and that these changes have likely been accompanied by additional gene regulatory changes affecting COX5A expression levels. Furthermore, we have suggested that these changes could affect aerobic energy metabolism among members of this group, in part because COX5Ap is a target of the thyroid hormone $\mathrm{T}_{2}$ [32]. In addition, a recent analysis of cellular scaling in the neocortex of anthropoids showed that elevated glia-neuron ratios correlate with brain enlargement [39], pointing to the role of glia in providing metabolic support to neurons requiring greater energy consumption for function. Because the anthropoid amino acid changes are located in the mitochondrial matrix, close to coevolving sites in subunits 2 and 4 (Table 2) proposed to have a COX regulatory function [29], the COX5Ap amino acid replacements observed in this study may have promoted better energy regulation in an increasingly high demand environment. While alternative interpretations of the data are possible - that, for instance, the higher rate of nonsynonymous substitutions in COX5A in anthropoids may be a function of their larger body and smaller presumed effective population size [40], which would reduce the effectiveness of purifying selection on slightly deleterious amino acid replacements [41]; or that the changed composition of mtDNA in anthropoids [42] may be the "motor" driving the putative positive selection of nuclear-encoded ETC [42], we note that COX5Ap amino acid sequences are identical in mammals with a wide range of body sizes and basal metabolic rates (e.g., shrew, cow), and that, within the context of intergenomic interactions between nuclear- and mitochondrially encoded ETC genes, it has been shown that rapid evolution in mitochondrially encoded subunits can indeed be associated with rapid divergence in interacting subunits [43] and that positive selection is particularly pronounced among interacting residues in ETC proteins $[15,44]$. More broadly, given that some of the main phenotypic differences associated with anthropoid origins are related to aerobic energy metabolism (e.g., longer lifespan, larger neocortex, and prolonged fetal development), both structural and regulatory evolutionary change in electron transport chain genes are likely to have contributed to the 
emergence and evolution of anthropoid primates $[8,9,24,45]$.

\section{Methods DNA sequences}

We sequenced the COX5A coding region for 16 mammalian species, including eight apes: human (Homo sapiens (2X)), common chimpanzee (Pan troglodytes), bonobo (Pan paniscus), gorilla (Gorilla gorilla), orangutan (Pongo pygmaeus), red-cheeked gibbon (Nomascus gabriellae) and siamang (Symphalangus syndactylus); two Old World monkeys: mantled guereza (Colobus guereza) and olive baboon (Papio anubis); three New World monkeys: pygmy marmoset (Callithrix pygmaea), white-lipped tamarin (Saguinus labiatus) and white-eared titi monkey (Callicebus donacophilus); three strepsirrhines: slow loris (Nycticebus coucang), brown greater galago (Otolemur crassicaudatus) and brown lemur (Eulemur fulvus) and one marsupial, the parma wallaby (Macropus parma) via RACE-PCR [33]. Four additional primate and two outgroup sequences were obtained from public databases: Philipine tarsier (Tarsius syrichta, AY 236506), common squirrel monkey (Saimiri sciureus, AY 585857), rhesus monkey (Macaca mulatta, AY_585861), human (NM_004255), mouse (Mus musculus, NM 007747), and cow (Bos Taurus, NM 00100289). Finally, 8 additional outgroup species were obtained from the ENSEMBL public database, including: frog (Xenopus tropicalis, ENSXETT00000045679), chicken (Gallus gallus, ENSGALT00000022855), gray short-tailed opossum (Monodelphis domestica, ENSMODT00000011691), African elephant (Loxodonta africana, ENSLAFT00000006266), cat (Felis catus, ENSFCAT00000010590), little brown bat (Myotis lucifugus, ENSMLUT00000013702), shrew (Sorex araneus, ENSSART00000003977) and rabbit (Oryctolagus cuniculus, ENSOCUT00000003256). Species in which there was more than one copy of COX5A, or for which poor reads were available, were not included in the study. All sequences newly obtained in this study were deposited in GenBank (DQ987236-DQ987252). Human reference sequence NM 004255 was used in the sequence analyses described below.

RNA was isolated either with the phenol-chloroform method or the RNeasy ${ }^{\oplus}$ midi kit (Qiagen, Valencia, CA). RACE PCR [33] was performed on these RNAs using the following primers: Pri_R1, 5' GGTATCATCAGTATTTCCAGG 3'; Pri_R2, 5' TTAAGTTGGTCTAAGTTCCTGG 3'; Pri_R3, 5' CTTATGAGGTCCTGCTTTGTC 3'; Apes_R1, 5' TTTGTCAAGGCCCAGTTCCTC 3'; Apes_R2, 5' GAGTGGA GATTCCCAGTTCATT 3'; Apes_R3, 5' TTGGTCTAAGTTCCTGGATGAC 3'; 5AF1, 5' CATGGGTCACANGAGCANGA 3'; 5AF2, 5' GAGTTTGATGCTCGCTGGGT 3'; 5AF3， 5' GCCAGAYATNGATGCYTGGGA 3 '. RACE PCR conditions were: 30 cycles of denaturation, $94^{\circ} \mathrm{C}, 40 \mathrm{~s}$; annealing, $57^{\circ} \mathrm{C}, 30 \mathrm{~s}$; and extension $72^{\circ} \mathrm{C}, 1 \mathrm{~min}$ and $10 \mathrm{~s}$; initial denaturation at $94^{\circ} \mathrm{C}$ for $2 \mathrm{~min}$ and a final extension at $72^{\circ} \mathrm{C}$ for $7 \mathrm{~min}$. RACE PCR products were visualized on $1 \%$ agarose gels and products were extracted using the Qiaquick $^{\circledast}$ (Qiagen, Valencia, CA) gel extraction kit. DNA sequencing reactions were performed using the Big Dye ${ }^{\circledast}$ (Applied Biosystems, Foster City, CA) cycle sequencing kit according to the manufacturer's specifications.

\section{Sequence analyses}

Phylogenetic relationships used to investigate patterns of COX5A nucleotide and amino acid sequence evolution were obtained from the literature $[46,47]$. Sequences were aligned using ClustalX 1.83.1 [48]. The PAML 3.15 [19] package was used to investigate the signature of positive selection among specific lineages and sites in the phylogeny. First, to investigate if rate heterogeneity existed among lineages, we compared a one omega $(\mathrm{dN} / \mathrm{dS})$ model (M0), which assumes the same relative rate (nonsynonymous divided by synonymous rate) for all lineages, with the free ratio model (M1), which allows rates to vary freely among the branches. Second, to investigate if the omega ratio varied among particular branches of interest in the phylogeny, we compared two different branch models. In the first model, two omega ratios were specified, one for all placental mammals (as a total group) and another for the remaining branches in the tree (i.e., the background). In the second model, three omega ratios were specified, one for the anthropoid clade (as a total group), another for the non-anthropoid placental mammals, and another for the remaining branches in the tree (i.e., the background). Finally, because all of these models assume a homogeneous omega ratio across codons, we also applied a branch-site test of positive selection on the anthropoid clade $[49,50]$. Model A (branch-site test of positive selection) assumes four classes of sites, the first two classes having omega values $0<\omega_{0}<1$ and $\omega_{1}=1$, assuming purifying selection and neutrality, respectively. The other two classes have the same assumption for the background branches, but allow for positive selection (i.e. $\omega_{2}>1$ ) on the foreground branches. This model was compared with the same model $\mathrm{A}$, but fixing the last two site classes to 1 according to Zhang et al. [50]. In all cases we checked for the existence of multiple local optima, running all models three times, with three different starting omega values $(0.5,1$, and 2$)$. Models were compared using the likelihood ratio test.

In addition to the previous model-based analyses, we reconstructed ancestral COX5A coding region nucleotide sequences to infer the amino acid changes that occurred among the sampled vertebrate lineages. Sequences were reconstructed, translated, and compared by three different methods: a maximum parsimony framework using ACCTRAN and DELTRAN algorithms as implemented in PAUP* [51], and the codon substitution model [52] 
implemented in PAML 3.15 [19]. Finally, to identify potential interactants with the COX5Ap amino acid replacements that occurred during anthropoid evolution, coding region nucleotide sequences were reconstructed, translated and compared for COX2p and COX4p (i.e., the genes encoding COX subunits in closest proximity to COX5Ap) using a maximum parsimony framework (ACCTRAN and DELTRAN). Ancestral reconstruction of COX4p sequences were based on analyses of sequences from Wildman et al. [16]. Ancestral reconstruction of COX2p sequences were based on analyses of sequences listed in Additional file 1. Proximity of potentially interacting residues in COX2p and COX4p was determined based on the crystal structure of cytochrome $c$ oxidase 2oCC, using Swiss-PDB viewer to identify the replacements occurring at a distance of $10 \AA$ or less.

\section{Real time PCR}

To examine and potentially validate the pattern of COX5A upregulation in humans reported in Uddin et al. [24], a SYBR green-based quantitative real-time quantitative PCR (qRT-PCR) strategy was designed to test the same individuals and species originally investigated by the microarray experiments: Homo sapiens $(\mathrm{N}=3)$, Pan troglodytes $(\mathrm{N}=1)$, Gorilla gorilla $(\mathrm{N}=1)$ and Macaca mulatta $(\mathrm{N}=3)$. cDNA was synthesized using total RNA (50 ng) obtained from the anterior cingulate cortex of each individual using the Stratascript $t^{\oplus}$ First-Strand Synthesis System from Stratagene (La Jolla, CA) by following manufacturer's protocols. A serial dilution of reference cDNA was also amplified for use in standard curve construction and as a calibrator for subsequent real-time experiments using Stratagene's qRTPCR Human Reference Total RNA (100 ng). COX5A-specific primers were designed in regions that were $100 \%$ conserved among the species and individuals hybridized to the microarray: COX5AF2 (5'-ATCCAGTCAGTTCGCTGCTA-3') and COX5AR2 (5'GTTTATCCCTTTACGCAATTCC-3') amplified a 123-bp product. COX5A expression levels were scaled to that of EIF3S7, a control gene on the Affymetrix HG-U133A and HG-U133B microarray chips that demonstrated one of the lowest standard deviations in expression levels among all tested samples (data not shown). Primers were designed in regions that were 100\% conserved among species with available genomic data: EIF3F1 (5'-AATGTGTTTGCCACTGATGC-3') and EIF3R1 (5'AGGAGGTCAAAGTCAGAGTTGTC-3') amplified a 137-bp product. For both primer sets, one primer was designed to fall in two exons in order to reduce the possibility of amplifying products from potentially contaminating genomic DNA. All primers were HPLC purified, and optimal concentrations were established using serial dilutions of reference cDNA in preliminary experiments.

Standard curves for both primer sets produced efficiencies $>91 \%$ and Rsq $>0.99$, thus meeting or exceeding the manufacturer's recommended values for ensuring a robust qRT-PCR assay. Quantitative RT-PCR reactions were run on Stratagene's Mx3000P instrument in triplicate for each and primer set on each individual and on each no template control (NTC) using the FullVelocity ${ }^{\circledR}$ SYBR $^{\circledast}$ Green QPCR Master Mix ${ }^{\circledast}$ (Stratagene) following manufacturer's protocols and including both the optional diluted reference dye and $0.25 \mu \mathrm{l}$ of AmpErase ${ }^{\circledast}$ Uracil $N$ glycosylase from Applied Biosystems (Foster City, CA). After ensuring that all NTCs were negative and that all samples fell within the linear range of amplification as previously determined by the standard curves, relative COX5A expression levels were calculated using the $2^{-\Delta \Delta \mathrm{C}} \mathrm{T}$ method described in Livak and Schmittgen [53] for target and reference samples, amplified in separate wells.

\section{Immunohistochemistry}

The localization of COX5Ap was examined in neocortex samples from non-geriatric adult macaque monkeys (Macaca maura), gorillas, common chimpanzees, and humans ( $\mathrm{N}=2$ individuals per species). Macaque monkey brains were obtained from animals transcardially perfused with $4 \%$ paraformaldehyde as part of an unrelated study. Brains of gorillas, chimpanzees, and humans were collected within 14 hours of death and immersed in 10\% formalin. Tissue sections were cut at a thickness of $40 \mu \mathrm{m}$ with a freezing microtome.

Immunohistochemistry was carried out on sections from the dorsolateral prefrontal cortex. Initial single-label immunohistochemical staining was performed using two different antibodies raised against the human COX5Ap, a mouse monoclonal IgG antibody (A-21363; Molecular Probes; dilution 1:200) and a rabbit polyclonal IgG antibody (11448-1-AP; Proteintech Group, Inc.; dilution 1:50). Prior to immunostaining, sections were pretreated for antigen retrieval by incubation in $10 \mathrm{mM}$ sodium citrate buffer $\left(\mathrm{pH} \mathrm{3.5)}\right.$ at $37^{\circ} \mathrm{C}$ in an oven for 30 minutes. Sections were then incubated in the primary antibody diluted in PBS with $2 \%$ normal serum and $0.1 \%$ Triton X100 detergent for approximately 48 hours on a rotator at $4{ }^{\circ} \mathrm{C}$. After rinsing in PBS, sections were incubated in a 1:200 dilution of Alexa Fluor 488-conjugated anti-mouse or anti-rabbit secondary antibody. Sudan Black B (1\% in $70 \%$ ethanol) was used to quench the autofluorescence generated by lipofuscin pigments.

To characterize further the cellular localization of COX5Ap, we also performed double-labeling immunofluorescence experiments in the perfusion-fixed macaque monkey samples using the polyclonal COX5Ap antibody in combination with mouse monoclonal antibodies against (1) microtubule-associated protein 2 (MAP2) (MAB3418, clone AP20; Chemicon International; dilution 1:200), (2) neuron-specific nuclear protein (NeuN) (MAB377; Chemi- 
con International; dilution 1:100), and (3) glial fibrillary acidic protein (GFAP) (MU020-UC; BioGenex Laboratories, Inc.; dilution 1:10,000). Both primary antibodies were incubated with the tissue simultaneously for 48 hours as described above. Immunoreactivity was revealed using an Alexa Fluor 488-conjugated anti-rabbit secondary antibody to visualize COX5Ap and an Alexa Fluor 568-conjugated anti-mouse secondary antibody to visualize MAP2, NeuN, and GFAP. In all immunohistochemistry runs, specificity of the reaction was confirmed by processing negative control sections excluding the primary antibody. No immunostaining was observed in control sections.

Brain sections were imaged with a BioRad MRC 1024 confocal laser scanning system coupled to an Olympus IX-70 microscope. Images were acquired with a $40 \times$ objective lens, a slice thickness of $1.5 \mu \mathrm{m}$, and a $z$ step of $0.5 \mu \mathrm{m}$. Figures show a $z$ projection of three contiguous confocal slices.

Quantification of the percentage of COX5Ap-immunoreactive cells that also contained NeuN in macaques $(n=2)$ was performed using the optical disector method as described previously [39] implemented in StereoInvestigator software (MBF Bioscience, Williston, VT). In two sections for each monkey, optical disector frames $(30 \mu \mathrm{m}$ $\times 30 \mu \mathrm{m} \times 6 \mu \mathrm{m}$ ) were placed within a contour drawn around the entire cortical width in the dorsolateral prefrontal cortex (Brodmann's area 9). Optical disectors were placed within this contour in a systematic random fashion using the fractionator method. Counting frames were investigated with a $63 \times$ objective (Zeiss Plan-Apochromat, N.A. 1.4) and cells were marked if their nucleolus was contained within the three-dimensional limits of the counting frame. Our estimate of the percentage of COX5A+NeuN-immunoreactive cells is based on a total sample of 619 cells.

\section{Authors' contributions}

JCO, MU, DEW, and CCS carried out the experimental work and data analysis, MG, and LIG carried out data analysis, and JCO, MU, DEW, CCS, PRH, MG, and LIG contributed to manuscript preparation and provided conceptual framework. All authors read and approved the final manuscript

\section{Additional material}

\section{Additional file 1}

Species name and Accession number of COII sequences used in this study. The listed taxa and associated COII sequences are the data used to determine the ancestral reconstructions of COX $2 p$ sequences reported in the text and in Table 2.

Click here for file

[http://www.biomedcentral.com/content/supplementary/14712148-8-8-S1.doc]

\section{Acknowledgements}

This work was supported by NIH grant ROI DK56927, NSF grants BCS03 I 8375, BCS0550209 BCS05 I 5484, and BCS0549 I I7, the WennerGren Foundation for Anthropological Research, and the James S. McDonnell Foundation (22002078). The authors thank Ms. Jennifer Leith and CheryI D. Stimpson for laboratory assistance, Dr. Maik Hüttemann for useful discussions and Dr. A. Popratiloff for assistance with confocal microscope imaging. For primate samples we thank Dr. Caro-Beth Stewart (SUNY, Albany), the Center for Reproduction of Endangered Species at the San Diego Zoological Society, Duke University Primate Center, Southwest National Primate Research Center, and Coriell Institute for Medical Research. The brain samples used in this study were loaned by the Great Ape Aging Project (USPHS/NIH grant AG I 4308, "A Comparative Neurobiology of Aging Resource," J. Erwin, PI), the Foundation for Comparative and Conservation Biology, and the Northwestern University Alzheimer's Disease Center Brain Bank (NADC grant P30 AGI 3854).

\section{References}

I. Adkins RM, Honeycutt RL: Evolution of the primate cytochrome c oxidase subunit II gene. J Mol Evol 1994, 38(3):2|5-23I.

2. Adkins RM, Honeycutt RL, Disotell TR: Evolution of eutherian cytochrome c oxidase subunit II: heterogeneous rates of protein evolution and altered interaction with cytochrome c. Molecular biology and evolution 1996, I3(10): | 393-1404.

3. Andrews TD, Easteal S: Evolutionary rate acceleration of cytochrome c oxidase subunit I in simian primates. J Mol Evol 2000, 50(6):562-568.

4. Andrews TD, Jermiin LS, Easteal S: Accelerated evolution of cytochrome $b$ in simian primates: adaptive evolution in concert with other mitochondrial proteins? J Mol Evol 1998, 47(3):249-257.

5. Baba ML, Darga LL, Goodman M, Czelusniak J: Evolution of cytochrome $C$ investigated by the maximum parsimony method. I Mol Evol I98I, I7(4): 197-2।3.

6. Doan JW, Schmidt TR, Wildman DE, Uddin M, Goldberg A, Huttemann M, Goodman M, Weiss ML, Grossman LI: Coadaptive evolution in cytochrome c oxidase: 9 of 13 subunits show accelerated rates of nonsynonymous substitution in anthropoid primates. Mol Phylogenet Evol 2004, 33(3):944-950.

7. Goldberg A, Wildman DE, Schmidt TR, Huttemann M, Goodman M, Weiss ML, Grossman LI: Adaptive evolution of cytochrome c oxidase subunit VIII in anthropoid primates. Proc Natl Acad Sci USA 2003, 100(10):5873-5878.

8. Grossman LI, Schmidt TR, Wildman DE, Goodman M: Molecular evolution of aerobic energy metabolism in primates. Mol Phylogenet Evol 200I, 18(I):26-36.

9. Grossman LI, Wildman DE, Schmidt TR, Goodman M: Accelerated evolution of the electron transport chain in anthropoid primates. Trends Genet 2004, 20(II):578-585.

10. Mishmar D, Ruiz-Pesini E, Mondragon-Palomino M, Procaccio V, Gaut B, Wallace DC: Adaptive selection of mitochondrial complex I subunits during primate radiation. Gene 2006, 378: I I-I8. 
I I. Osada N, Kusuda J, Hirata M, Tanuma R, Hida M, Sugano S, Hirai M, Hashimoto K: Search for genes positively selected during primate evolution by 5 '-end-sequence screening of cynomolgus monkey cDNAs. Genomics 2002, 79(5):657-662

12. Schmidt TR, Goodman M, Grossman Ll: Molecular evolution of the COX7A gene family in primates. Molecular biology and evolution 1999, I6(5):619-626.

13. Schmidt TR, Goodman M, Grossman LI: Amino acid replacement is rapid in primates for the mature polypeptides of $\mathrm{COX}$ subunits, but not for their targeting presequences. Gene 2002 286(I): 13-19.

14. Schmidt TR, Jaradat SA, Goodman M, Lomax MI, Grossman LI: Molecular evolution of cytochrome $c$ oxidase: rate variation among subunit Vla isoforms. Molecular biology and evolution 1997 | 4(6):595-60 I.

15. Schmidt TR, Wildman DE, Uddin M, Opazo JC, Goodman M, Grossman LI: Rapid electrostatic evolution at the binding site for cytochrome c on cytochrome c oxidase in anthropoid primates. Proc Natl Acad Sci USA 2005, I 02( I 8):6379-6384.

16. Wildman DE, Wu W, Goodman M, Grossman LI: Episodic positive selection in ape cytochrome c oxidase subunit IV. Molecular biology and evolution 2002, 19(10): 1812-1815

17. Wu W, Schmidt TR, Goodman M, Grossman LI: Molecular evolution of cytochrome c oxidase subunit I in primates: is there coevolution between mitochondrial and nuclear genomes? Mol Phylogenet Evol 2000, I 7(2):294-304.

18. Rizzuto R, Nakase H, Zeviani M, DiMauro S, Schon EA: Subunit Va of human and bovine cytochrome c oxidase is highly conserved. Gene 1988, 69(2):245-256.

19. Yang Z: PAML: a program package for phylogenetic analysis by maximum likelihood. Comput Appl Biosci 1997, I3(5):555-556.

20. Yang Z: Computational Molecular Evolution. Oxford: Oxford University Press; 2006

21. Golding GB, Dean AM: The structural basis of molecular adaptation. Mol Biol Evol I998, I5(4):355-369.

22. Goodman M: Positive selection causes purifying selection. Nature 1982, 295:630.

23. Ortlund EA, Bridgham JT, Redinbo MR, Thornton JW: Crystal structure of an ancient protein: evolution by conformationa epistasis. Science 2007, 3 I 7(5844): I544-I548.

24. Uddin M, Wildman DE, Liu G, Xu W, Johnson RM, Hof PR, Kapatos G, Grossman LI, Goodman M: Sister grouping of chimpanzees and humans as revealed by genome-wide phylogenetic analysis of brain gene expression profiles. Proc Natl Acad Sci USA 2004, I 0 I(9):2957-2962.

25. Initial sequence of the chimpanzee genome and comparison with the human genome. Nature 2005, 437(7055):69-87.

26. Gibbs RA, Rogers J, Katze MG, Bumgarner R, Weinstock GM, Mardis ER, Remington KA, Strausberg RL, Venter JC, Wilson RK, Batzer MA, Bustamante CD, Eichler EE, Hahn MW, Hardison RC, Makova KD, Miller W, Milosavljevic A, Palermo RE, Siepel A, Sikela JM, Attaway T, Bell S, Bernard KE, Buhay CJ, Chandrabose MN, Dao M, Davis C Delehaunty KD, Ding $Y$, et al:: Evolutionary and biomedical insights from the rhesus macaque genome. Science 2007 , 3 I 6(5822):222-234.

27. Karaman MW, Houck ML, Chemnick LG, Nagpal S, Chawannakul D Sudano D, Pike BL, Ho VV, Ryder OA, Hacia JG: Comparative analysis of gene-expression patterns in human and African great ape cultured fibroblasts. Genome Res 2003, I3(7): I619-1630.

28. Sherwood CC, Hof PR: The evolution of neuron types amd cortical histology in apes and human. In The Evolution of Primate Nervous Systems Volume 4. Edited by: Preuss TM, Kaas JH. Oxford: Academic Press; 2007:355-378.

29. Hüttemann M, Kadenbach $B$, Grossman LI: Mammalian subunit IV isoforms of cytochrome c oxidase. Gene 200 I, 267(I): I I I-I 23.

30. Goglia F: Biological effects of 3,5-diiodothyronine (T(2)). Biochemistry (Mosc) 2005, 70(2): 164-172.

31. Horst C, Rokos H, Seitz HJ: Rapid stimulation of hepatic oxygen consumption by 3,5-di-iodo-L-thyronine. Biochem J 1989 26 I (3):945-950.

32. Arnold S, Goglia F, Kadenbach B: 3,5-Diiodothyronine binds to subunit $\mathrm{Va}$ of cytochrome-c oxidase and abolishes the allosteric inhibition of respiration by ATP. Eur J Biochem 1998 252(2):325-330.

33. Wrutniak-Cabello C, Casas F, Cabello G: Thyroid hormone action in mitochondria. J Mol Endocrinol 200I, 26(I):67-77.
34. Sheehan TE, Kumar PA, Hood DA: Tissue-specific regulation of cytochrome c oxidase subunit expression by thyroid hormone. Am J Physiol Endocrinol Metab 2004, 286(6):E968-974.

35. de Escobar GM, Obregon MJ, del Rey FE: Maternal thyroid hor mones early in pregnancy and fetal brain development. Best Pract Res Clin Endocrinol Metab 2004, I 8(2):225-248.

36. Kester $\mathrm{MH}$, Martinez de Mena R, Obregon MJ, Marinkovic D Howatson A, Visser TJ, Hume R, Morreale de Escobar G: lodothyronine levels in the human developing brain: major regulatory roles of iodothyronine deiodinases in different areas. Clin Endocrinol Metab 2004, 89(7):31 I7-3128.

37. Morreale de Escobar G, Obregon MJ, Escobar del Rey F: Role of thyroid hormone during early brain development. Eur J Endocrinol 2004, I 5 I (Suppl 3):U25-37

38. Gagneux P, Amess B, Diaz S, Moore S, Patel T, Dillmann W, Parekh $R$, Varki A: Proteomic comparison of human and great ape blood plasma reveals conserved glycosylation and differences in thyroid hormone metabolism. Am J Phys Anthropol 2001, I I 5(2):99-109.

39. Sherwood CC, Stimpson CD, Raghanti MA, Wildman DE, Uddin M Grossman LI, Goodman M, Redmond JC, Bonar CJ, Erwin JM, Hof PR: Evolution of increased glia-neuron ratios in the human frontal cortex. Proc Natl Acad Sci USA 2006.

40. Popadin K, Polishchuk LV, Mamirova L, Knorre D, Gunbin K: Accumulation of slightly deleterious mutations in mitochondrial protein-coding genes of large versus small mammals. Proc Natl Acad Sci USA 2007, I 04(33): I 3390-I 3395.

4I. Ohta T: Synonymous and nonsynonymous substitutions in mammalian genes and the nearly neutral theory. J Mol Evol 1995, 40(I):56-63

42. Schmitz J, Ohme M, Zischler $\mathrm{H}$ : The complete mitochondria sequence of Tarsius bancanus: evidence for an extensive nucleotide compositional plasticity of primate mitochondrial DNA. Mol Biol Evol 2002, I 9(4):544-553.

43. Willett CS, Burton RS: Evolution of interacting proteins in the mitochondrial electron transport system in a marine copepod. Molecular biology and evolution 2004, 2 I (3):443-453.

44. Schmidt TR, Wu W, Goodman M, Grossman LI: Evolution of nuclear- and mitochondrial-encoded subunit interaction in cytochrome c oxidase. Mol Biol Evol 200I, I 8(4):563-569.

45. Caceres M, Lachuer J, Zapala MA, Redmond JC, Kudo L, Geschwind $\mathrm{DH}$, Lockhart DJ, Preuss TM, Barlow C: Elevated gene expression levels distinguish human from non-human primate brains. Proc Natl Acad Sci USA 2003, I 00(22): I 3030-I3035.

46. Goodman M, Porter CA, Czelusniak J, Page SL, Schneider H, Shoshan J, Gunnell G, Groves CP: Toward a phylogenetic classification of Primates based on DNA evidence complemented by fossil evidence. Mol Phylogenet Evol 1998, 9(3):585-598.

47. Murphy WJ, Eizirik E, O'Brien SJ, Madsen O, Scally M, Douady CJ, Teeling E, Ryder OA, Stanhope MJ, de Jong WW, Springer MS: Resolution of the early placental mammal radiation using Bayesian phylogenetics. Science 200I, 294(5550):2348-235 I.

48. Thompson JD, Gibson T], Plewniak F, Jeanmougin F, Higgins DG: The CLUSTAL_ $X$ windows interface: flexible strategies for multiple sequence alignment aided by quality analysis tools. Nucleic Acids Res 1997, 25(24):4876-4882.

49. Yang Z, Nielsen R: Codon-substitution models for detecting molecular adaptation at individual sites along specific lineages. Mol Biol Evol 2002, I 9(6):908-917.

50. Zhang J, Nielsen R, Yang Z: Evaluation of an improved branchsite likelihood method for detecting positive selection at the molecular level. Molecular biology and evolution 2005 22( I 2):2472-2479.

51. Swofford D: PAUP* phylogenetic analysis using parsimony (*and other methods). In 4.0b/O Sunderland, MA: Sinauer Associates; 2002

52. Goldman N, Yang Z: A codon-based model of nucleotide substitution for protein-coding DNA sequences. Mol Biol Evol I 994, I I(5):725-736.

53. Livak KJ, Schmittgen TD: Analysis of relative gene expression data using real-time quantitative PCR and the 2(-Delta Delta C(T)) Method. Methods 200I, 25(4):402-408 